\title{
Raising the Standard of Systematic Reviews Published in Environment International
}

Paul Whaley* (1), Robert J. Letcher (2), Adrian Covaci (3), Ruth Alcock (1)

(1) Lancaster Environment Centre, Lancaster University, Lancaster LA1 4YQ, UK

(2) Environment and Climate Change Canada, National Wildlife Research Centre, 1125 Colonel By Drive, Carleton University, Ottawa, ON, Canada

(3) Toxicological Centre, University of Antwerp, Universiteitsplein 1, 2610 Wilrijk, Belgium

A well-reported study provides sufficient information about its methods to either allow the reader to attempt to reproduce its findings or, since one cannot rush to the laboratory to replicate every published paper, at least gives the reader the opportunity to evaluate the credibility of its results.

Evidence syntheses, i.e. the papers which attempt to combine the results of multiple individual studies into an overall bottom-line result, should be no different. As for primary studies, these manuscripts should provide clear and unambiguous descriptions of their methods, including but not limited to: their research objectives; how they found the literature they reviewed; how they appraised the validity of this literature; a justification of their techniques for statistical analysis; and their approach to characterising the strength of the evidence for their conclusions.

The purpose of an evidence synthesis is to discover what existing evidence is saying in answer to a particular research question. Such "studies of studies" contribute to scientific progress by making sense of what is often a contradictory and methodologically heterogeneous body of research, helping distinguish research results which are most likely true from those which are not, and hence that which is known from that which is not.

As for primary research, not all evidence syntheses are equally rigorous: in medicine, widespread problems with the reporting quality of evidence syntheses have been recognised for at least three decades (e.g. Mulrow 1987; McAlister et al. 1999; Klimo et al. 2014). In contrast, hard data on the reporting quality of evidence syntheses in environmental health research is very limited. What there is, is worrying. For example, a recent review of current practice in conducting evidence syntheses, undertaken as part of a systematic review of the developmental toxicity of perfluorinated octanoic acid (Woodruff, Sutton 2014), is suggestive of widespread shortcomings in the reporting of methods used (Figure 1).

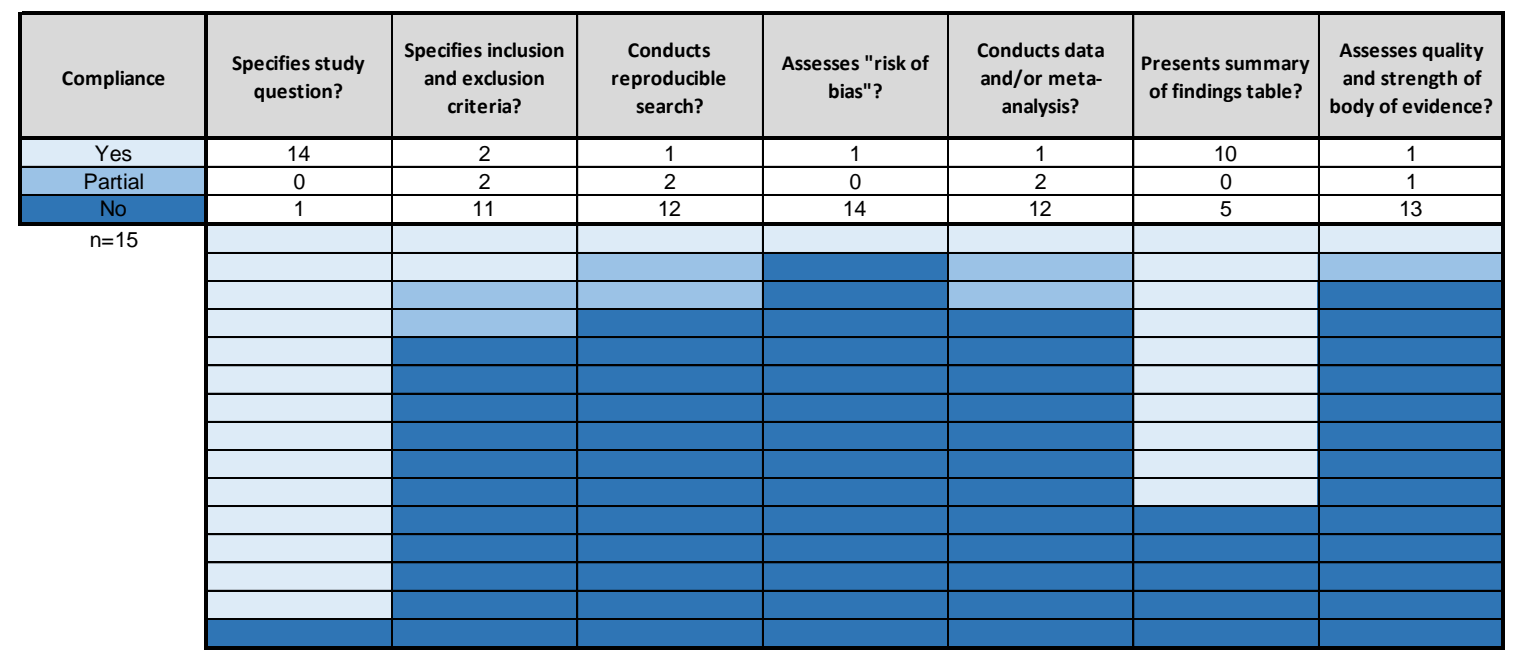

[Figure 1: "Comparison of the PFOA reviews' methods according to key features of Cochrane and GRADE systematic and transparent review methods." Adapted from Woodruff \& Sutton (2014), Table 1] 
The "systematic review" is one potential remedy to addressing shortcomings in the conduct and reporting of methods used in synthesising evidence in environmental health research. First developed in medicine and the social sciences, a systematic review is a transparent, reproducible, and consistent approach to minimising risk of bias in identifying, appraising and summarising evidence of the effects of a medical, public health or social intervention - or, more commonly in environmental health research, exposure to an environmental agent (Whaley et al. 2016). Interest in systematic review methods is now flourishing in environmental health, with exponential growth in manuscripts at least claiming to be systematic reviews (Figure 2). It is therefore a good time, if not overdue, for Environment International to introduce new reporting standards and editorial processes which will help ensure that all systematic reviews and related methods papers which are published in the journal are of the highest achievable quality.

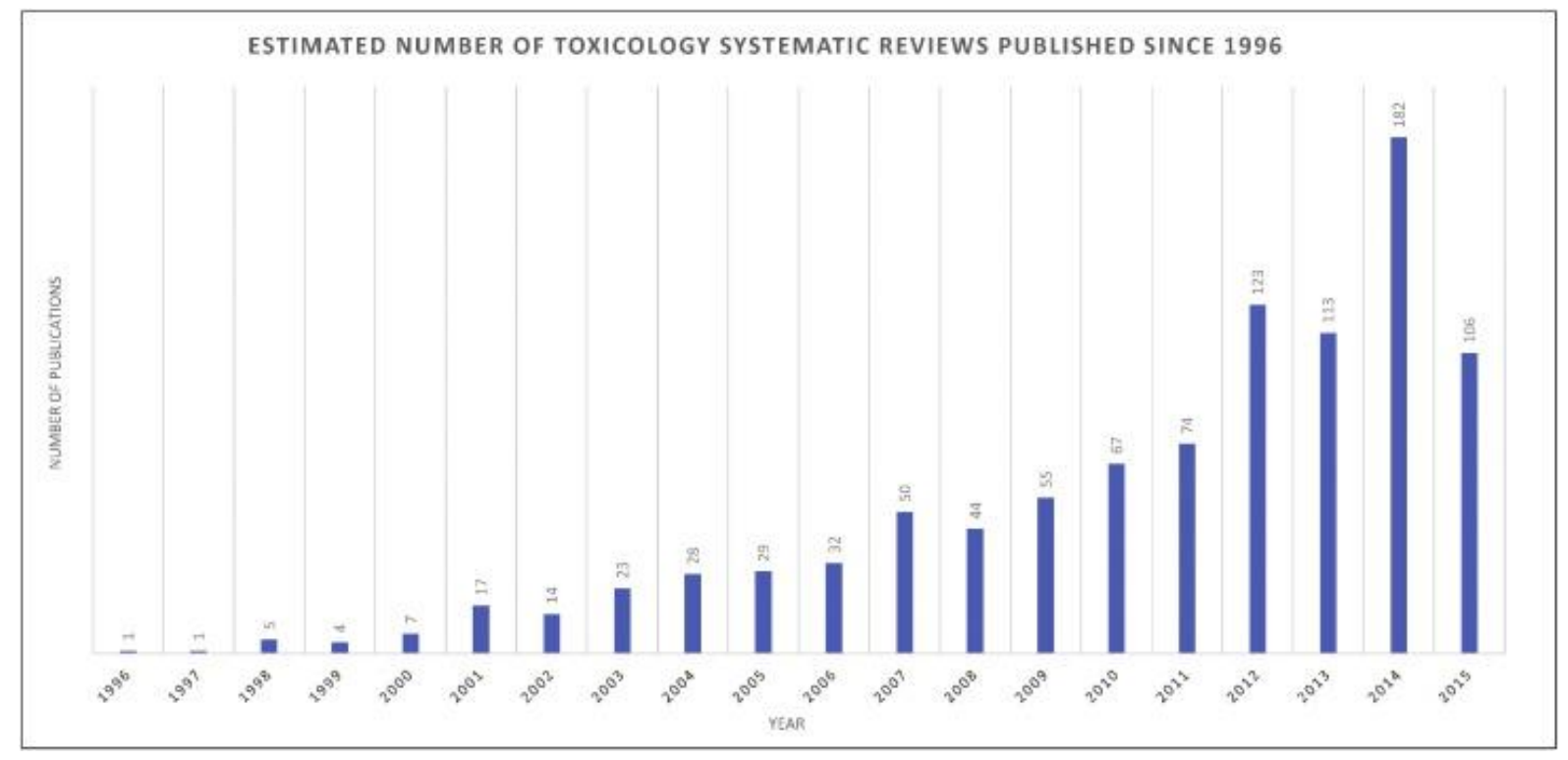

[Figure 2: Papers indexed in Web of Science (WoS) with the term "Systematic Review" in the publication title, filtered for "Toxicology" as topic, excluding topic of "Pharmacology Pharmacy". WoS database search excludes Biosis Citation Index (not subscribed). Date of search: 4 April 2016. Reproduced from Whaley \& Halsall (2016).]

The present challenge is in choosing an editorial intervention which will be effective. To inform its decisions, Environment International has been able to draw on recent experience of publishing the first-ever issue of an environmental health journal dedicated to systematic reviews (Whaley \& Halsall 2016), and as a first step has appointed a dedicated "Associate Editor for Systematic Reviews" with a specific remit to ensure it publishes only high-quality methods papers and systematic reviews worthy of the title (Environment International 2016).

A second step has been to endorse the PRISMA Statement (Moher et al. 2009), a widely-used checklist of items for authors to include when reporting a systematic review or meta-analysis. While 179 academic journals have endorsed PRISMA, Environment International appears, at time of writing, to be only the second environmental health journal to do so (PRISMA 2016).

The inquisitive reader will, however, soon discover that reporting guidelines may do little to improve reporting standards in the published literature: for example, a recent systematic review reported found little evidence for the efficacy of endorsement of reporting guidelines as an intervention for improving reporting quality (Stevens et al. 2014). The lesson, however, is not necessarily that guidelines such as PRISMA have no value, rather that implementation has to be emphasised over endorsement in order to see a beneficial effect on publishing standards.

Unlike many journals, adherence to the PRISMA standard for authors submitting systematic reviews to Environment International is therefore compulsory rather than voluntary: all new systematic review manuscripts submitted to the journal must be accompanied by a completed PRISMA checklist 
(see supplemental information) explaining how the submission fulfils each checklist item or, in the event an item cannot be fulfilled, justification as to why this is the case.

A third step has been to improve the peer-review process for submitted systematic review manuscripts, by inviting where feasible two systematic review experts and two topic experts per submission. Finally, in addition to the PRISMA checklist, which already provides valuable structure and information to reviewers of systematic reviews, a fourth step is to develop formal guidance and training for peer-reviewers on reviewing systematic review manuscripts submitted to Environment International, in order to ensure comprehensive, exacting and fair review of all submitted manuscripts. This is currently under development.

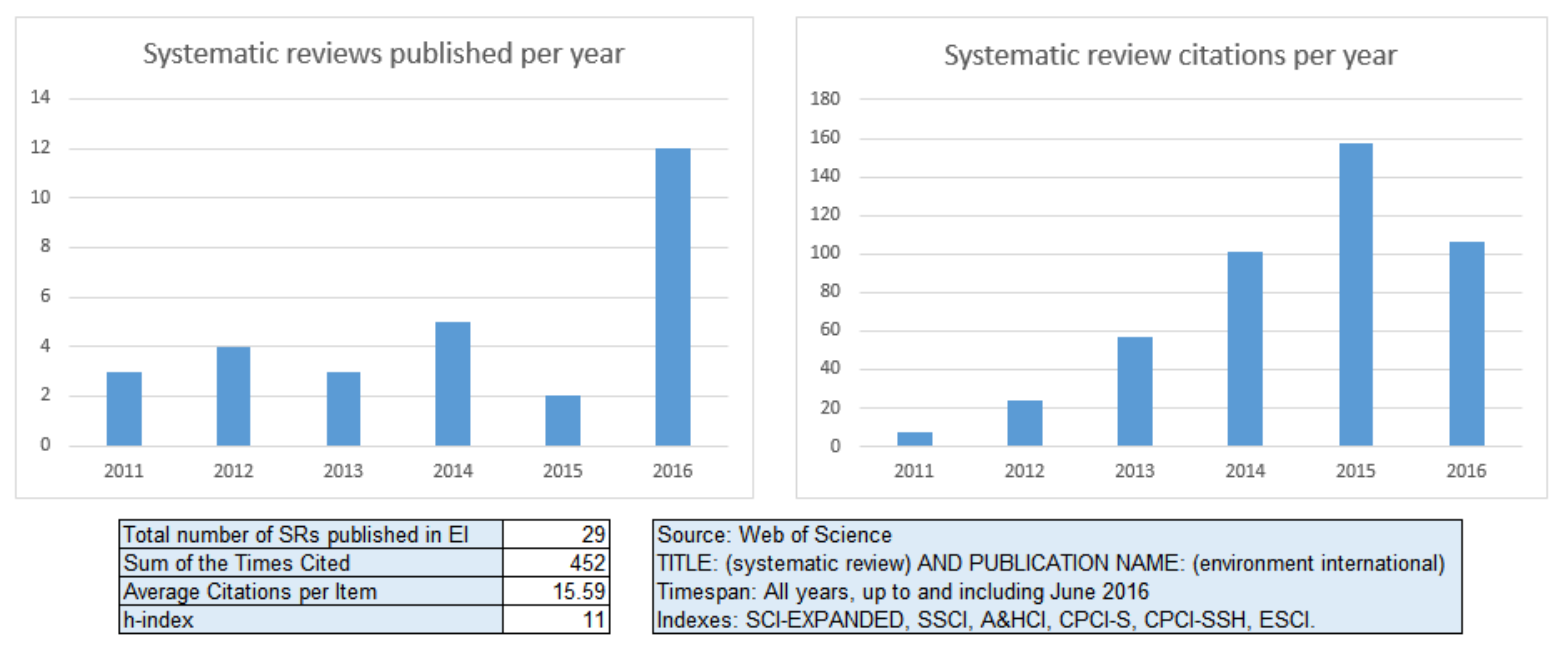

[Figure 3: Data on systematic reviews published in Environment International]

Prospective authors should view these measures not as a barrier to submission, but as guidance and encouragement to polish submitted systematic reviews. Manuscripts which can demonstrate compliance with a high standard and have been subjected to comprehensive and exacting peerreview will be better-received and, it is hoped, produce more reliable results, which should ultimately be reflected in higher impact and greater numbers of citations. Peer-reviewers will see benefits as well: because they will only dealing with submissions which have been pre-screened for quality, there will be less time wasted on reviewing manuscripts with obvious and critical methodological shortcomings such as insufficiently reported research objectives, search strategies, statistical techniques, etc.

Environment International is already a leading journal in the field of environmental health, attracting increasing numbers of increasingly-cited systematic reviews (Figure 3). By implementing these standards, the journal hopes to attract ever-better publications from ambitious scientists, continuing to turn this cycle of improvement and help our authors contribute to the ever-growing understanding of environmental health science.

\section{Publication bibliography}

Environment International (2016): Paul Whaley joins as Associate Editor for Systematic Reviews.

Elsevier B.V. Available online at http://www.journals.elsevier.com/environment-

international/news/paul-whaley-joins-as-associate-editor-for-systematic-reviews, checked on $8 / 8 / 2016$.

Klimo, Paul; Thompson, Clinton J.; Ragel, Brian T.; Boop, Frederick A. (2014): Methodology and reporting of meta-analyses in the neurosurgical literature. In Journal of neurosurgery 120 (4), pp. 796-810. DOI: 10.3171/2013.11.JNS13195. 
McAlister, F. A.; Clark, H. D.; van Walraven, C.; Straus, S. E.; Lawson, F. M.; Moher, D.; Mulrow, C. D. (1999): The medical review article revisited: has the science improved? In Annals of internal medicine 131 (12), pp. 947-951.

Moher, David; Liberati, Alessandro; Tetzlaff, Jennifer; Altman, Douglas G. (2009): Preferred reporting items for systematic reviews and meta-analyses: the PRISMA statement. In Annals of internal medicine 151 (4), p. 264-9, W64. DOI: 10.1371/journal.pmed.1000097.g001.

Mulrow, C. D. (1987): The medical review article: state of the science. In Annals of internal medicine 106 (3), pp. 485-488.

PRISMA (2016): PRISMA Endorsers. PRISMA. Available online at http://www.prismastatement.org/Endorsement/PRISMAEndorsers.aspx, checked on 8/8/2016.

Stevens, Adrienne; Shamseer, Larissa; Weinstein, Erica; Yazdi, Fatemeh; Turner, Lucy; Thielman, Justin et al. (2014): Relation of completeness of reporting of health research to journals' endorsement of reporting guidelines: systematic review. In BMJ (Clinical research ed.) 348, pp. g3804. DOI: 10.1136/bmj.g3804.

Whaley, Paul; Halsall, Crispin (2016): Assuring high-quality evidence reviews for chemical risk assessment: Five lessons from guest editing the first environmental health journal special issue dedicated to systematic review. In Environment international 92-93, pp. 553-555. DOI: 10.1016/j.envint.2016.04.016.

Whaley, Paul; Halsall, Crispin; Agerstrand, Marlene; Aiassa, Elisa; Benford, Diane; Bilotta, Gary et al. (2016): Implementing systematic review techniques in chemical risk assessment: Challenges, opportunities and recommendations. In Environment international 92-93, pp. 556-564. DOI: 10.1016/j.envint.2015.11.002.

Woodruff, Tracey J.; Sutton, Patrice (2014): The Navigation Guide systematic review methodology: a rigorous and transparent method for translating environmental health science into better health outcomes. In Environmental health perspectives 122 (10), pp. 1007-1014. DOI:

10.1289/ehp.1307175. 